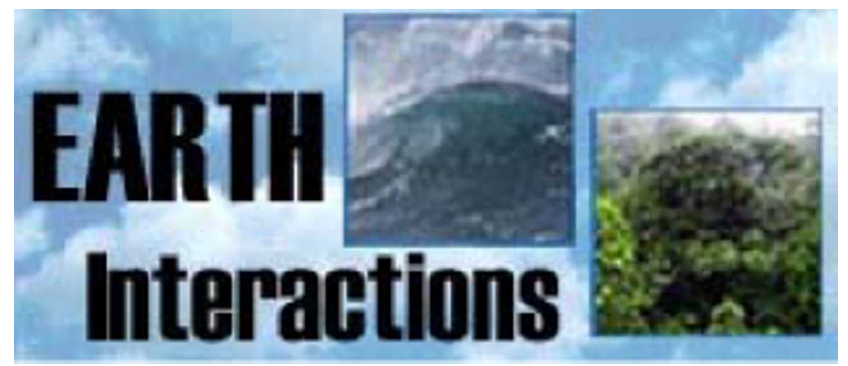

Copyright (c) 2005, Paper 09-000; 4,966 words, 7 Figures, 0 Animations, 2 Tables. http://EarthInteractions.org

\title{
Detection of Mesoscale Seasonal and Interannual Variation in the Vegetation of the Amazon Basin
}

\section{Miles G. Logsdon*}

Department of Oceanography, University of Washington, Seattle, Washington

\section{Robin Weeks and Milton Smith}

Department of Geology, University of Washington, Seattle, Washington

\section{Jeffery E. Richey}

Department of Oceanography, University of Washington, Seattle, Washington

\section{Victoria Ballester}

Brazilian Centro de Energia Nuclear y Agricultura, University of São Paulo, Piracicaba, São Paulo, Brazil

\section{Yosio Shimabukoro}

Instituto Nacional De Pesquisas Espaciais, Sao Jose dos Campos, São Paulo, Brazil Received 14 September 2004; accepted 9 August 2005

* Corresponding author address: Miles G. Logsdon, University of Washington, P.O. Box 355351, Seattle, WA 98195-5351.

E-mail address: mlog@u.washington.edu 
Earth Interactions - Volume 9 (2005) - Paper No. 25 - Page 2

\begin{abstract}
In the Amazon basin, seasonal and interannual spectral changes measured by satellites result from anthropogenic disturbance and from the interaction between climate variation and the surface cover. Measurements of spectral change, and the characterization of that change, provide information concerning the physical processes evident at this mesoscale. A 17-yr sequence of daily Advanced Very High Resolution Radiometer (AVHRR) global area coverage $(\mathrm{GAC})$ images were analyzed to produce a monthly record of surface spectral change encompassing El Niño-Southern Oscillation (ENSO) cycles. Monthly cloud-free composite images from daily AVHRR data were produced by linear filters that minimized the finescale spatial variance and allowed for a wide range analysis within a consistent mathematical framework. Here the use of a minimized local variance (MLV) filter that produced spatially smooth images in which major land-cover boundaries and spatial gradients are clearly represented is discussed. Changes in the configuration of these boundaries and the composition of the landscape elements they defined are described in terms of quantitative changes in landscape pattern. The time series produced with the MLV filter revealed a marked seasonal difference in the pattern of the landscape and structural differences over the length of the time series. Strikingly, the response of the region to drier El Niño years appears to be delayed in the MLV series, the maximum response being in the year following El Niño with little or no change seen during El Niño.
\end{abstract}

KEYWORDS: Remote sensing; Landscape change; Time series analysis

\title{
1. Introduction
}

Information on how the surface energy balance of the Amazon basin changes on seasonal and interannual time scales is crucial to understanding how the basin responds to climatic and, ultimately, anthropogenic forcing. The determination of spectral changes and their translation into biophysical properties over time in the Tropics, however, faces a number of difficulties. Daily remotely sensed data cannot be used directly to determine physical aspects of surface changes due to frequent cloud cover, smoke, and a variable atmosphere. However, these obfuscations of the surface can be filtered from a longer time series dataset of images. The energy balance of the land surface is a product of changes in intercepted radiation, vegetation cover, phenology, and surface water balance, all of which may in turn affect the spectral signature of the surface. Thus, changes in the surface spectral signature can be indicative of changes in the biogeochemical and hydrological systems.

We address issues related to the observation and interpretation of surface change by first creating a time series of monthly Advanced Very High Resolution Radiometer (AVHRR) composite images using a finite impulse response (FIR) filter to minimize local variation (MLV) in the spectral signature of pixels through both space and time (Figure 1). We then calculate a series of simple spatial pattern metrics that characterize the spatial composition and configuration of the general land-cover change in the basin. This approach is applied to a 17-yr time series of images of the northern half of the South American continent. The question we are asking is, What is the spatial pattern of seasonal and interannual variability in the spectral response of the land surface? 
Earth Interactions - Volume 9 (2005) - Paper No. 25 - Page 3

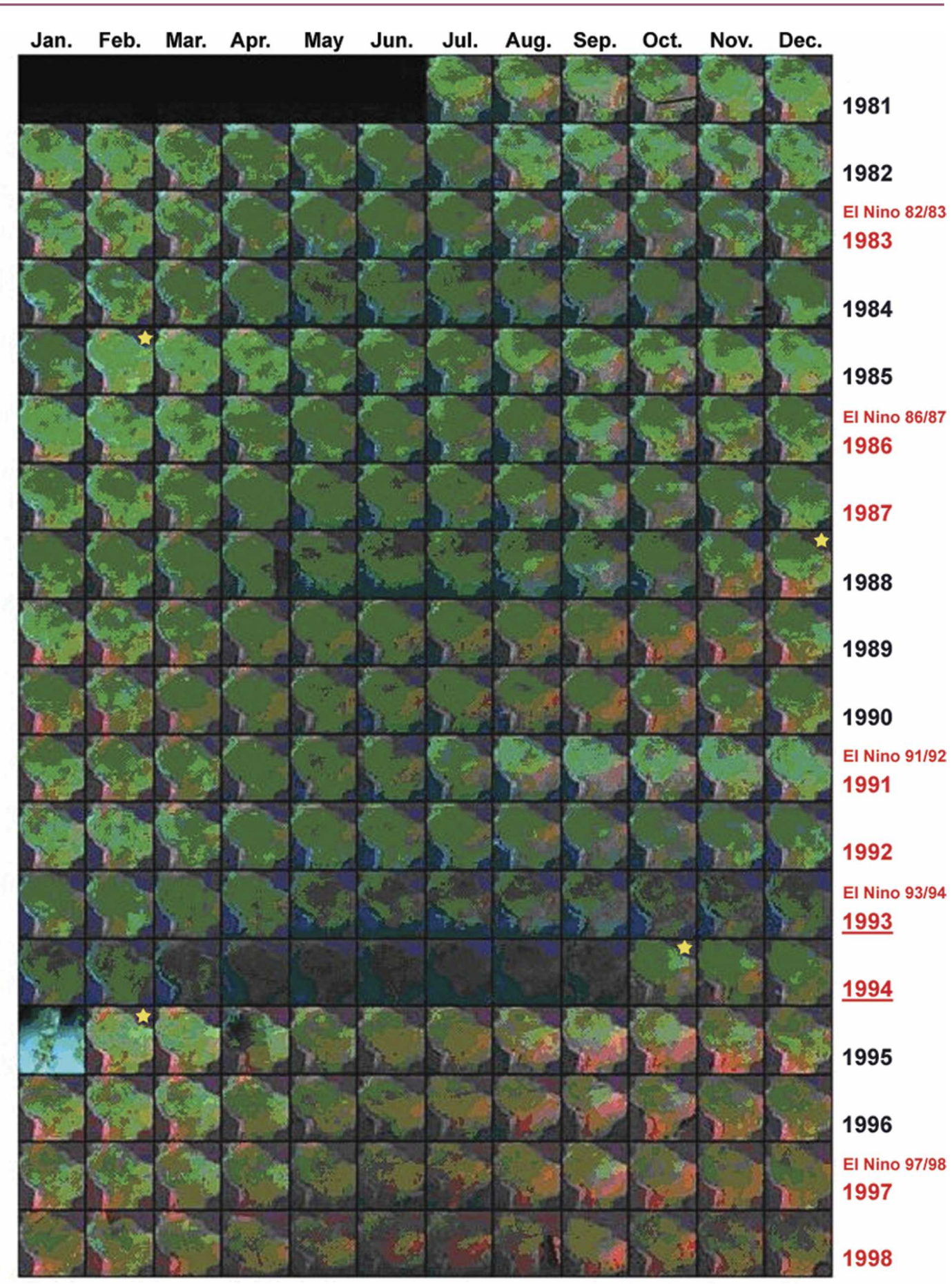

Change in the satellite instrument series.

Figure 1. AVHRR monthly cloud-free color composites using the MLV filter in the spectral signature of channels 1 (red), 2 (green), and 3 (blue) for pixels of daily images through both space and time. The yearly sequences with El Niño condition are noted along with changes in the AVHRR instruments. 
In the landscape of the Amazon basin, the mature tropical forest and savannas form a landscape matrix, or backdrop, upon which patches of distinct land-cover types reside. The spatial pattern of these different landscape elements and their changes through time are products of both cultural and natural processes.

AVHRR data have been used in a number of investigations of Amazon rainforest vegetation and deforestation. Setzer and Pereira (Setzer and Pereira 1991) used 46 images to investigate biomass burning within the basin. Tucker et al. (Tucker et al. 1984) and Malingreau and Tucker (Malingreau and Tucker 1988) have monitored the extent of deforestation and forest clearing in the regions of Rondonia, Acre, and Mato Grosso, Brazil. In general, these investigations have suggested that the spatial resolutions of AVHRR data will underestimate small areas of deforestation and create errors in land-cover classification; yet due to the high temporal frequency of image acquisition, the data are well suited for monitoring vegetation responses at coarse spatial scales. Again, this emphasizes the importance of an approach that explicitly addresses both the spatial and temporal scale of the information contained in the remotely sensed dataset.

We recognize the Amazon basin as a landscape composed of ever-changing elements. The spatial "scale" at which those elements are recognized or defined, and the temporal scale that corresponds to measured changes, are central issues in a line of research that now integrates remote sensing (RS) and geographic information systems (GIS) analysis. A quantitative description of the basin's changing spatial structure is sometimes referred to as landscape physiognomy or landscape pattern analysis (Dunning et al. 1992; Turner et al. 1989). In this type of analysis it is useful to distinguish between measures of composition and configuration in the description of either the variety and abundance of patches (composition), or the shape and relative placement of those patches (configuration) for the landscape as a whole. Together these indices describe the spatial structure of the landscape and are not statistical inferences for spatial process (see Ripley 1988; Cressie 1991). We have chosen descriptive measures of composition and configuration to compare over time because of our interest in describing the pattern and fragmentation of the homogeneous elements in the Amazon basin as a whole.

\section{Methods}

The MLV method that we used for this investigation selects pixels from daily images that minimize the temporal average of local spatial variability over a monthly time period. The rationale for this approach is that the largest physical source of spatial and temporal variance is atmospheric variability, including clouds that are resolved and unresolved (i.e., subpixel), and the effects of viewing or illumination geometry. Although clouds cause variance at all spatial scales, it is the variance at the local scale (less than 10 pixels or approximately $40 \mathrm{~km}$ ) that is exploited in our analysis in order to provide the best separation between atmospheric variance and important mesoscale landscape variation. Minimized finescale spatial variance is achieved by selecting pixels using a filter that is determined empirically by its effect on our ability to visually interpret the spatial and temporal variance of a training dataset of mesoscale landscape units that included recognizable reservoirs, savannas, long-term deforestation activity, the river main steam, and the Pantanal (Figures 2 and 3). 


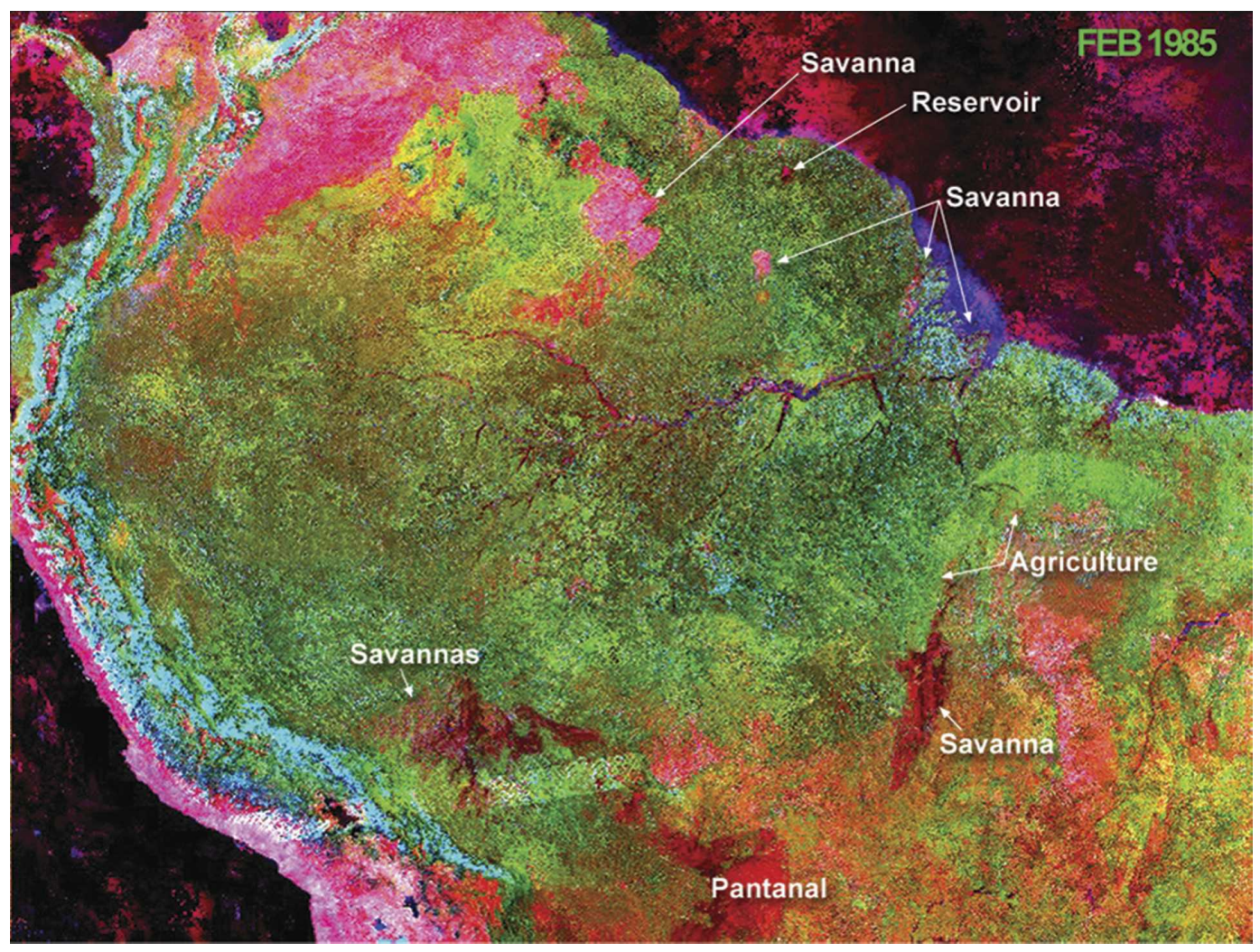

Figure 2. High contrast stretched color composite (channels 1 (red), 2 (green), and 3 (blue)) for visual interpretation of Feb 1985 training sites and reference areas used for empirical selection of MLV filters and as a decision tool for deletion of monthly images from the time series.

In quantifying changes in the spatial pattern at seasonal and interannual time scales, we first recognize that the characterization of pattern of any landscape is a function of scale. Ecological (or landscape) scale encompasses a description of both extent and grain (Turner et al. 1989; Wiens 1989). Therefore, the spatial extent (or range) of a process and the grain (resolution or size) of an individual observation define the ecological scale of a process. Recording location and describing a spatial relationship defines its pattern and structure. The aim of quantifying the spatial and temporal pattern of these basinwide datasets is to better describe not only the variety in composition but also the configuration of the changing elements within the basin. From this perspective we ensure that both the spatial and temporal scales of local variability in the time series dataset capture the anticipated heterogeneity of the mesoscale land forms. In addition, this approach ensures that metrics quantifying changes in the spatial pattern characterize seasonal and interannual responses of these landforms to functional changes.

AVHRR is a scanning radiometer instrument that records reflectance in five bands of the electromagnetic spectrum: channels $1(0.58-0.68 \mu \mathrm{m}), 2(0.72-1.1$ $\mu \mathrm{m}), 3(3.55-3.93 \mu \mathrm{m}), 4(10.3-11.3 \mu \mathrm{m})$, and $5(11.3-12.5 \mu \mathrm{m})$. The local area 


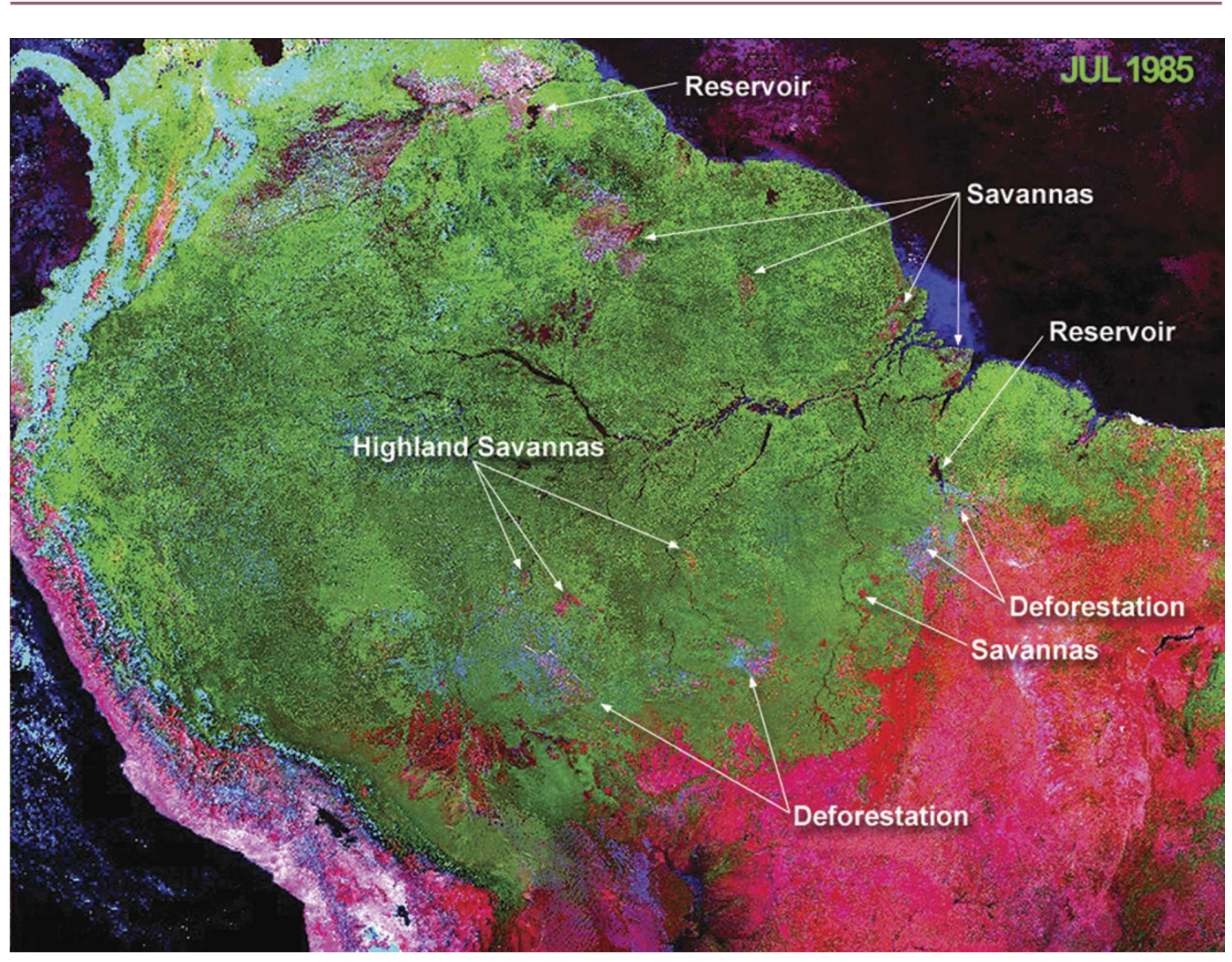

Figure 3. Same as in Fig. 2, but for Jul 1985.

coverage (LAC) of the instrument is $1.1-\mathrm{km}$ resolution and the global area coverage (GAC; the onboard averaged values of 16 LAC pixels) is about 4.4-km resolution. Tucker et al. (Tucker et al. 1984) used the reflectance sensitivity of channels 1 and 2 to monitor green leaf biomass. Channel 3 is at the border between the optical and thermal ranges with sensitivity to a combination of emitted and reflected radiation and surface temperatures.

The time series dataset used in this analysis comprises a 17-yr, daily sequence of AVHRR GAC images covering a period from July 1981 to December 1998 and spanning four satellites, NOAA-7, -9, -11, and -14 (Table 1). The multiple satellite passes for each day were reduced to a single daily coverage by selecting the pass with the smallest zenith angle. The embedded calibration coefficients provided for each band were applied to the data. Satellites changed from NOAA-7 to NOAA-9

Table 1. Satellites used in creating the time series dataset.

\begin{tabular}{lcc}
\hline Satellite & Start date & End date \\
\hline NOAA-7 & 25 Jun 1981 & 31 Jan 1985 \\
NOAA-9 & 1 Feb 1985 & 7 Nov 1988 \\
NOAA-11 & 8 Nov 1988 & 13 Sep 1994 \\
NOAA-9 & 14 Sep 1994 & 22 Jan 1995 \\
NOAA-14 & 23 Jan 1995 & 31 Dec 1998 \\
\hline
\end{tabular}


in February 1985 and from NOAA-9 to NOAA-11 in November 1988. The NOAA-9 satellite was used again beginning in September 1994 and changed to NOAA-14 in January 1995. Pixels judged to be from spectrally constant targets exhibited significant temporal drift in bands 1-3 for each of the satellites. In contrast, over a 9-yr calibrated image sequence from 1990 to 1998, channels 4 and 5 appeared to be stable to within $5 \%$ over the same period. The drift in bands $1-3$ is primarily due to drift in the satellite orbit (equator crossing time), which causes the average solar zenith angle for a given pixel location to increase with time for each satellite. Smaller effects are due to instrument drift. A first-order correction was applied to channels 1 and 2 to remove the drift associated with each satellite.

The daily image coverage is reduced to monthly cloud-free composite images. In the rainy season, complete cloud-free images over the Amazon basin are extremely rare. The atmosphere problem is exacerbated in the dry season by smoke. A common approach to making cloud-free monthly composite images is to select pixels with the maximum value of the normalized difference vegetation index (MNDVI) during each month (Goward et al. 1994; Justice et al. 1985; Sellers et al. 1994). Monthly composite images constructed in this way have become the database archive, and are assumed to represent the spectral and spatial signature of land cover. However, this optimization may allow artifacts, arising from viewing/ illumination geometry, calibration, atmosphere, and surface mixtures to propagate into the measurements of spectral change.

We applied a simple form of a FIR filter,

$$
\mathrm{Ci}=\sum_{b=1}^{N} w_{b} R_{b}+C,
$$

where $R_{b}$ are the reflectances in the spectral bands $b$, and $w_{b}$ are filter weights applied to those values, and $C$ is a constant. The filter is used to determine a cloud index (Ci) from the first 3 AVHRR bands (or channels; $\mathrm{ch}_{1}, \mathrm{ch}_{2}$, and $\mathrm{ch}_{3}$ ) as follows:

$$
\mathrm{Ci}=-\left(w_{1} \mathrm{ch}_{1}+w_{2} \mathrm{ch}_{2}+w_{3} \mathrm{ch}_{3}\right) .
$$

The cloud index is the output of the FIR filter; and $\mathrm{ch}_{1}, \mathrm{ch}_{2}$, and $\mathrm{ch}_{3}$ are the reflectances in the first three AVHRR channels. The method selects the pixel that minimizes the cloud index in any given month. The weights $(w)$ of the FIR filter are determined using training data together with a criterion in which a desired characteristic of the resulting composite images is evaluated. By cycling through all possible combinations of filter weights, applying the resulting filters as a cloud index for the training areas, and then evaluating the selection criteria for the resulting composites, we empirically determined the filter that provided the best visual interpretation of the mesoscale training area (Figures 2 and 3). For the purpose of this project we used filter weights that consistently selected pixels that minimized the temporal average of local spatial variability. Minimized finescale spatial variance was achieved by selecting pixels using a filter that was determined empirically, as described below, by its effect on the spatial and temporal variance of a training dataset:

$$
\mathrm{Ci}=-\left(\mathrm{ch}_{2}-0.01 \mathrm{ch}_{2} \mathrm{ch}_{2}\right) \text {. }
$$


Earth Interactions - Volume 9 (2005) - Paper No. 25 - Page 8

The final step in processing of the image sequence involved the application of a one-dimensional median wavelet filter (Donoho 1994) to each pixel in each channel separately in the temporal direction. This affected some of the fine detail in the images that had short temporal duration. Even though each pixel was filtered independently, a net visual effect on the images was a slightly smoother spatial appearance. It should be emphasized that no spatial smoothing was applied at any stage in the MLV approach.

The next step of our analysis began once the processing of the original AVHRR daily images into a monthly cloud-free time series of multispectral pixel values was completed. These pixel values were assigned to discrete spectral clusters using a two-step self-organizing procedure. For the time series as a whole, the spectral values were first assigned to spectral clusters using a standard Iterative Self Organizing Data Analysis Technique Algorithm (ISODATA) approach to determine 5 times the number of clusters of the desired final product. We sought to characterize the pattern of broadscale (or coarse) landscape process and, therefore, generated 40 initial clusters. These clusters were reclassed into the final eight clusters based upon two criteria: the ability to separate the spectral means of each cluster and the combination of clusters, which consistently allowed for a confident visual identification of the training or reference landscape units (Figures 2 and 3). This step generalized (smoothed) the spatial heterogeneity of each monthly image.

A number of the monthly images were still well outside the acceptable range for a visually consistent spatial comparison. These monthly images were removed from further analysis. Deleting these images from the monthly time series dataset was our primary means of quality control for comparing differences in the resulting spatial pattern metrics. In some observations, the spectral distances to the cluster means for recognized persistent mesoscale landscape units exceeded the threshold accounted for by anticipated spectral variation. In these observations, it appeared that either the MLV filter had failed to select appropriate pixel values or that instrument calibration errors, such as georegistration occurred. Since our goal was to investigate the spatial changes over a long time period, we eliminated monthly images where we could not, with confidence, identify the training or reference landscape units (Figures 2 and 3).

The resulting spectral signature for the cluster means was used to classify each monthly image using a nearest-neighbor algorithm. Regional groupings of similarly classed pixels that formed a minimum landscape patch size of 100 continuously connected pixels were preserved in the final landscape map, while smaller discontinuous patches were assigned to the landscape cluster classes with which they shared the longest border. The selection of the minimum patch size was based upon the consistent minimum size of the reference landscape units. Because the spectral range of each monthly image is potentially very different, the spectral clusters (patches) were not named or assigned consistent identifier throughout the time series. Each monthly landscape map was, therefore, a mosaic of unnamed classified spectral clusters (patches) of surface responses within the range of spectral responses throughout the complete time series (Figure 4).

In the final step, a suite of spatial pattern metrics were calculated for each classified monthly mean image to characterize the spatial composition and configuration of the individual months. These metrics and their ecological applications and limitations are described by McGarigal and Marks (1994). We relied on the 
Jan. Feb. Mar. Apr. May Jun. Jul. Aug. Sep. Oct. Nov. Dec.

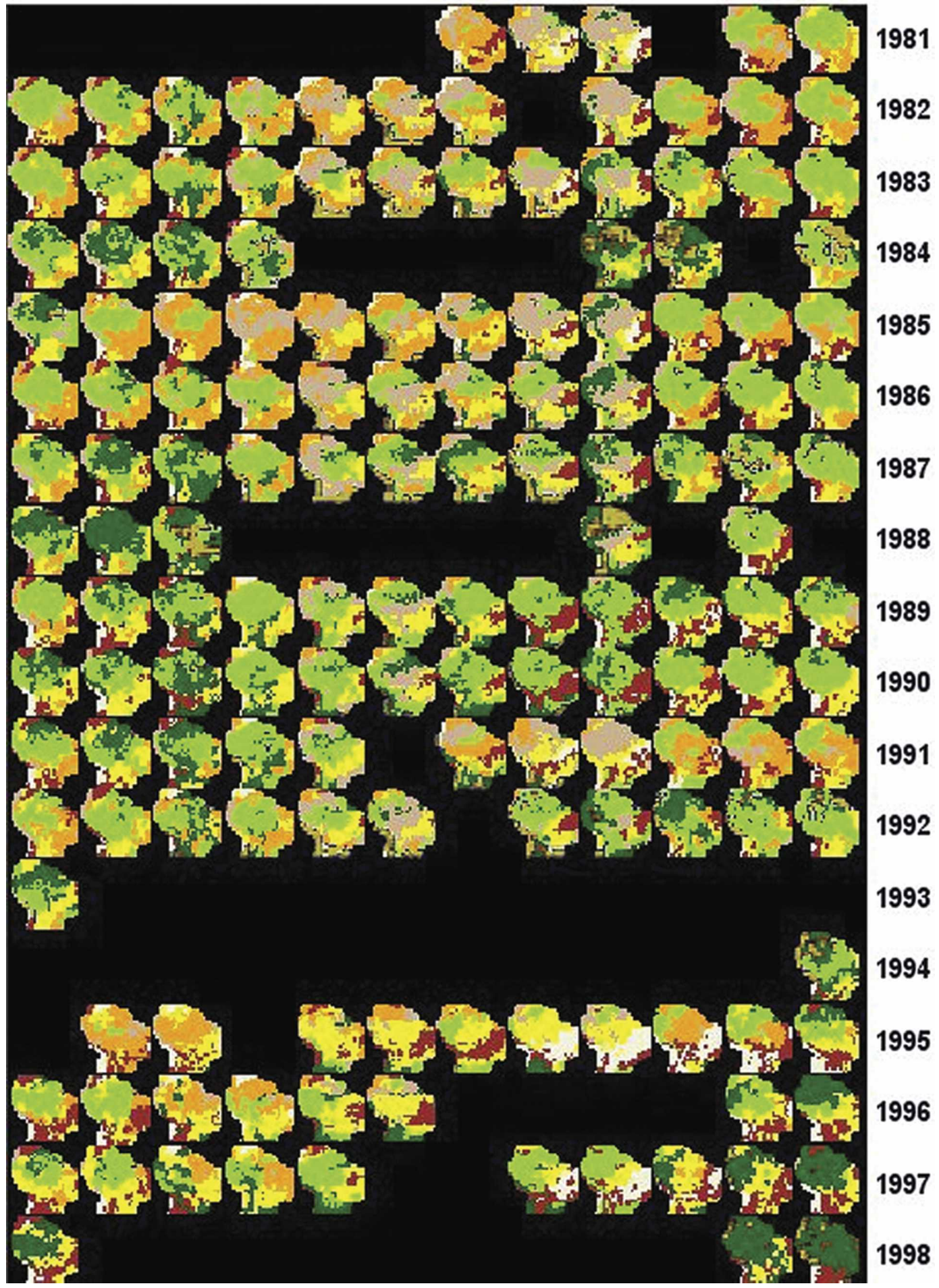

Figure 4. The regional groupings (patches) of similarly unnamed classed pixels that formed a minimum-sized landscape patch. 
Earth Interactions • Volume 9 (2005) - Paper No. 25 • Page 10

number of patches (NP) and the landscape patch index (LPI) to characterize basinwide fragmentation. Changes in the shape and complexity of the average landscape patch were described by changes in the landscape shape index (LSI) and mean patch fractal dimension (MPFD). The interspersion and juxtaposition index (IJI) was used to describe changes in the spatial configuration of patches by recording the percent of all possible shared edges between patches of different types. These metrics and associated coefficient of variance (the relative percent of the mean represented by the standard deviation) illustrate the composition and configuration complexity of the season and interannual change in the classified images (Tables 2a, 2b).

\section{Results}

Visual inspection of the monthly cloud-free time series images revealed many changes throughout the basin. Dry nonforest areas, including small savanna enclaves, and open-water bodies were all easily and consistently detected. Wet savannas were distinguishable from dry savannas and the primary forest. Seasonal

Table 2a. Average monthly landscape indices of NP, LPI, LSI, MPFD, and IJI.

\begin{tabular}{lccccc}
\hline Month & NP & LPI & LSI & MPFD & IJI \\
\hline Jan & 21.00 & 28.46 & 7.79 & 1.35 & 66.89 \\
Feb & 98.00 & 25.08 & 9.64 & 1.27 & 65.57 \\
Mar & 92.25 & 21.61 & 9.65 & 1.29 & 67.23 \\
Apr & 93.73 & 18.99 & 8.43 & 1.26 & 70.12 \\
May & 84.00 & 25.45 & 9.04 & 1.29 & 68.67 \\
Jun & 103.33 & 15.00 & 9.39 & 1.27 & 71.96 \\
Jul & 82.86 & 25.03 & 9.38 & 1.29 & 70.63 \\
Aug & 24.10 & 26.23 & 7.96 & 1.33 & 72.40 \\
Sep & 20.78 & 26.78 & 7.96 & 1.34 & 70.18 \\
Oct & 22.08 & 25.78 & 7.97 & 1.35 & 65.60 \\
Nov & 20.80 & 29.94 & 7.95 & 1.37 & 67.21 \\
Dec & 21.43 & 32.32 & 7.57 & 1.34 & 67.23 \\
\hline
\end{tabular}

Table $2 \mathrm{~b}$. Coefficient of variation in average monthly indices throughout the time series.

\begin{tabular}{lcrrrr}
\hline Month & NP & LPI & LSI & MPFD & IJI \\
\hline Jan & 17.0 & 8.6 & 10.3 & 2.6 & 10.1 \\
Feb & 19.1 & 6.4 & 11.9 & 1.8 & 6.5 \\
Mar & 76.9 & 29.8 & 13.9 & 3.9 & 8.6 \\
Apr & 22.2 & 25.7 & 12.3 & 1.7 & 5.7 \\
May & 53.1 & 9.4 & 11.2 & 4.2 & 9.0 \\
Jun & 13.2 & 15.6 & 8.2 & 2.0 & 3.4 \\
Jul & 51.8 & 10.2 & 11.8 & 4.1 & 7.4 \\
Aug & 11.8 & 25.8 & 14.3 & 1.1 & 6.0 \\
Sep & 12.0 & 28.9 & 9.7 & 1.9 & 8.1 \\
Oct & 12.7 & 19.8 & 13.0 & 2.0 & 7.2 \\
Nov & 17.4 & 19.3 & 13.8 & 1.8 & 8.0 \\
Dec & 22.8 & 24.6 & 16.3 & 3.2 & 8.2 \\
\hline
\end{tabular}


Earth Interactions • Volume 9 (2005) • Paper No. 25 • Page 11

green-up and dryout of the central Brazilian cerrado was detected and observed to change over time. Within the Amazon forest, large areas dominated by secondary forest, a large eucalyptus/pine plantation, and part of the bamboo-dominated forest were also detected. A seasonal leaf flush also seemed to be visible in the Roraima, Guyana, piedmont, and plains transition forest. Agriculture, pastures, and secondary forests along the occupied portion of the Transamazon Highway contributed to a consistent seasonal brightness in band 2 during the rainy seasons. The deforested crescent of the southern and southeastern edges of the Amazon sometimes appeared in the rainy season. Intensively deforested areas with only a small percentage of secondary forest were otherwise visible only in the dry season. Some expected features, spectrally very distinct in Landsat Thematic Mapper (TM) images, were not detected. The sandy-soil forests of the upper Rio Negro basin, much of the bamboo-dominated forest, and forests in floodplains at the Japurá/ Solimões, Brazil, confluence were difficult to distinguish.

One observation particularly suitable for this time series was the dominant climatic effect of ENSO conditions at the basin scale. The maximum observed effect on surface vegetation was in the years following El Niño. This was seen as a decrease in the albedo (inferred from AVHRR $\mathrm{ch}_{1}$ and $\mathrm{ch}_{2}$ reflectance) of the terra firme and, for the large savannas, as a delay in the usual rise of $\mathrm{ch}_{3}$ at the start of the dry season. Although calibration effects in the MLV-filtered data may have affected the interpretation of the response to El Niño, there was little visible vegetation response coincident with El Niño events until the following year. This was most easily observed in the 1987/88 and 1991-94 sequences.

The seasonal and annual spatial changes in the clustered data demonstrated a clear difference between the wet and dry seasons. The location of the edge between patches of differing surface responses along the southern boundary of the basin varied greatly during the onset of the dry season (February) and then again at the beginning of the wet season, occurring approximately in June (Figure 5).

Through visual inspection alone, the patterns of the changing landscape were difficult to understand. A review of relative change in pattern metrics designed to characterize the seasonal and interannual variation in the spatial composition and configuration of spectral clusters offered a quantitative tool for expressing change. A general reduction in the patchiness of the landscape occurred throughout the season as the average NP for each month ranged from a low of 21 in January to a high of 103 in June (Table 2a). Likewise, the LPI demonstrated an average dry season landscape more dominated by larger and fewer patches in December $(\mathrm{LPI}=32)$ than the more fragmented and smaller patch size of the average June (LPI $=15$; see Figures 6 and 7). The configuration of these patches revealed relatively little change in the distribution of observed adjacency between cluster types. The IJI revealed little change in the distribution of adjacencies among unique patch types. As the number of landscape patches increased and decreased over the seasons, the distribution of patches (or cluster types) adjacent to each other remained relatively even $(65.57>$ IJI < 72.40; Table 2a; Figure 6).

These seasonal patterns remained evident as the analysis was extended to characterize the whole time series. A total of 54 out of the 210 monthly images were omitted from the pattern analysis due to poor georegistration or because the spectral clusters means for some spatial cluster fell well outside the class limits. This made a comparison of all months for all years throughout the time series unreli- 


\section{Southern Boundary of Spectral Clusters of Common Surface Response}

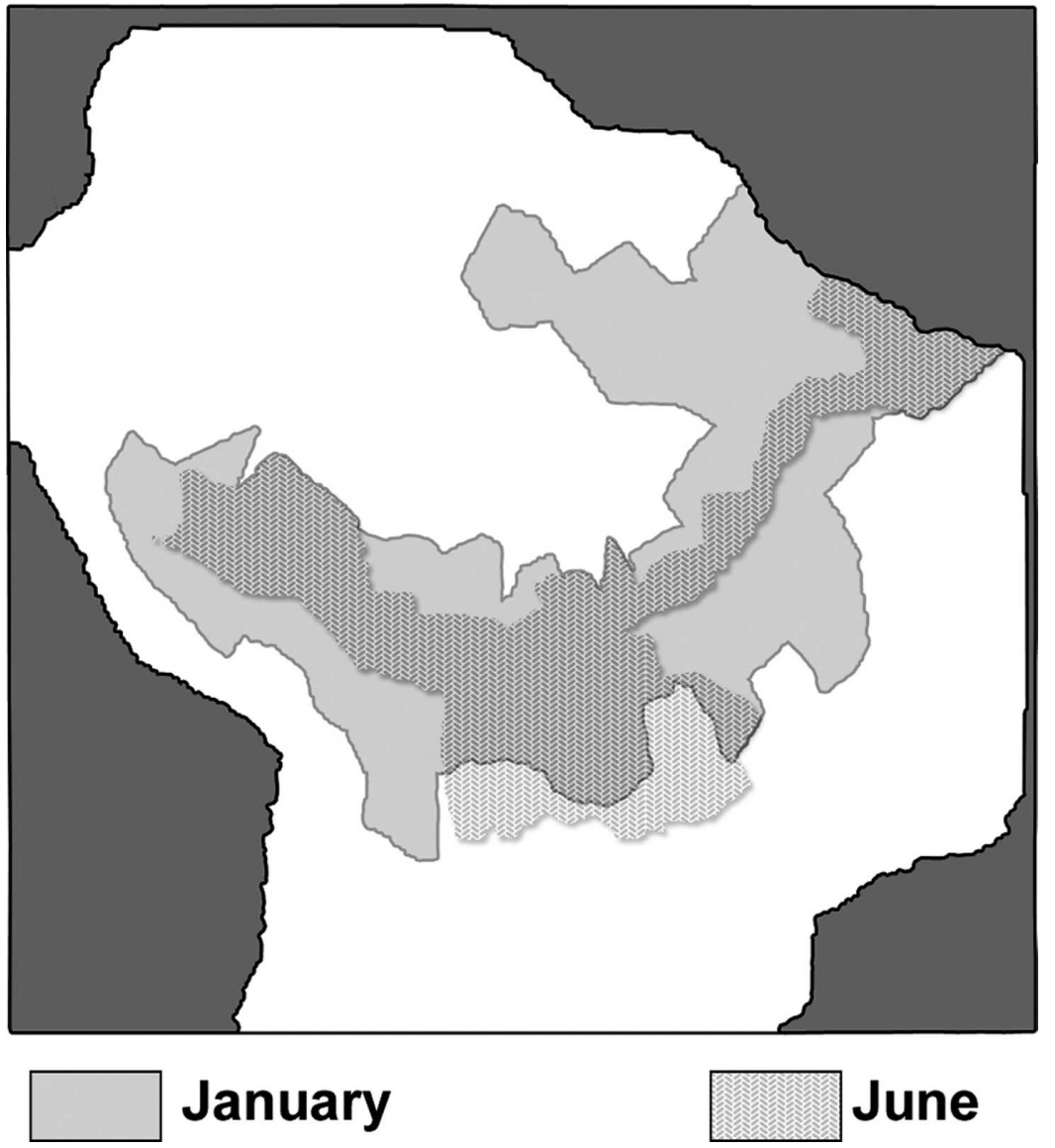

Figure 5. Seasonal locations of the edge between patches of differing surface response along the southern boundary of the basin.

able. Comparison of the variability in the landscape pattern metrics for those months which were used in this analysis illustrates that some months are more consistent in their landscape response than others (Table 2b). The interannual variability in the complexity of the pattern for the months of May and June indicate a very dynamic shift occurs at this time. The month of May is more variable in the number of patches and their adjacency, yet more consistent in the size and complexity of shape. We have illustrated the change in spatial patterns over the 17-yr time series by comparing the months of January, April, June, August, and October for the years 1982, 1983, 1986, 1987, 1989, 1990, 1991, 1992, and 1997 (Figures 6 and 7). The 1989 and 1990 images had the fewest number of patches during the wet season while the dry season remained relatively unchanged. The remaining landscape pattern metrics illustrate a varying landscape. The LPI reveals an increase in the dominance of larger patches later in the time series for most months. 


\section{Landscape Metrics}

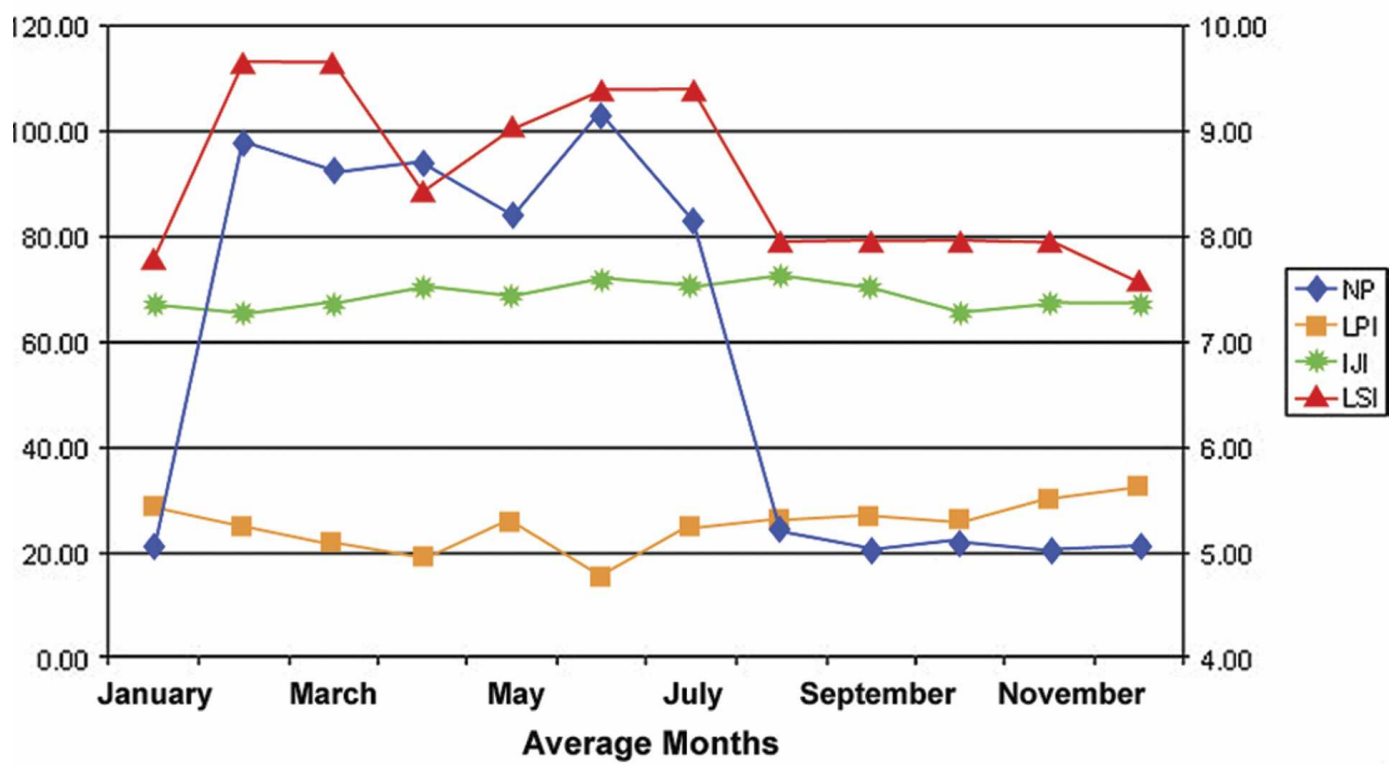

Figure 6. Seasonal variation in landscape metrics for the average month. The composition metrics of the NP and LSI indicate seasonal differences in the landscape's fragmentation, while the configuration metrics of LPI and IJI remain stable.

\section{Conclusions}

All methods of producing cloud-free composite images introduce bias into the resulting image sequences. Given the number of variables that can affect spectral data measured at the satellite, there is always a trade-off between precision in detection of surface change and immunity to effects from extrinsic (undesired) factors. Decisions that are made with respect to this trade-off affect the extendibility of image interpretation in space and time. Filters such as MLV, that are sensitive to viewing/illumination geometry, produce images whose pixels are selected from a less variable and nearer nadir set of geometries. These considerations affect the magnitude of estimates of surface change observed in seasonal cycles.

We have characterized the visible and quantitatively descriptive changes in the pattern of the Amazon basin over a 17-yr monthly time series. At this scale we visually observed a delayed vegetation response to climatic changes associated with El Niño as an indication of changes in the interannual pattern of the surface response. These observations seemed inconsistent with biosphere model assumptions that suggested that changes in vegetation were coincident with climatic change, yet may have been consistent with phonological states triggered by seasonal and longer climatic events such as El Niño. While the landscape pattern metrics indicate no clear trends in fragmentation at this spatial resolution, the metrics do reveal interannual variation suggestive of responsiveness to mesoscale events such as flooding or drought. Laurance and Williamson (Laurance and 

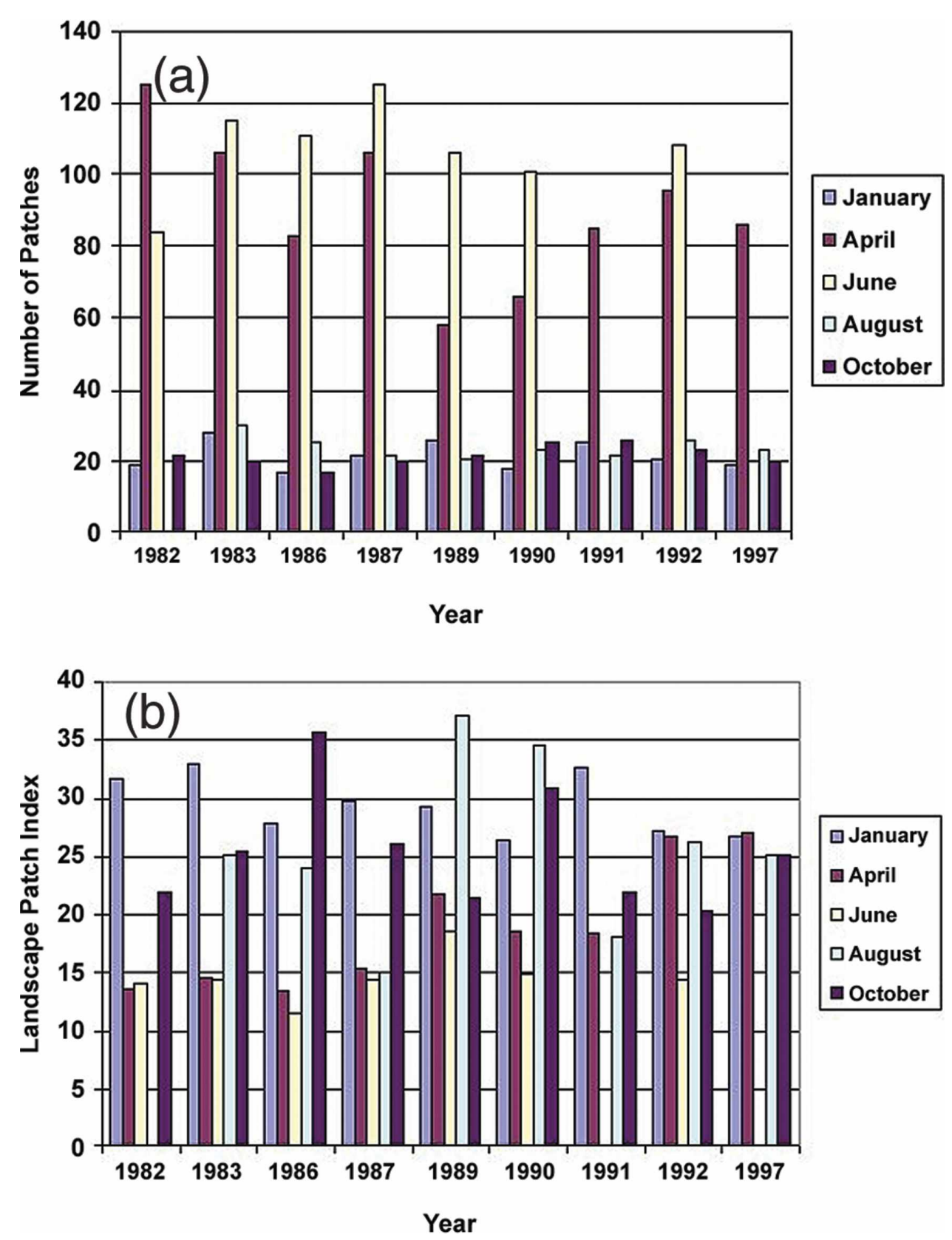

Figure 7. Seasonal and interannual change in landscape pattern metrics of (a) NP and (b) LPI.

Williamson 2001) observed that the fragmented forests of the Amazon basin are more prone than intact forests to periodic damage from ENSO droughts and "positive feedbacks among forest loss, fragmentation, fire, and regional climate change appear increasingly likely." Likewise, the 1979-99 land surface dataset developed by Dirmeyer and Tan (Dirmeyer and Tan 2001) for specifying initial 
Earth Interactions • Volume 9 (2005) • Paper No. 25 • Page 15

and/or boundary conditions for modeling applications of the Amazon basin produced realistic climatology while also indicating significant interannual variability.

This analysis characterized the seasonal and interannual variation in the Amazon basin. The basin was shown to exhibit structural changes at the landscape scale on both seasonal and interannual cycles. Our goal was to describe the spatial pattern of seasonal and interannual variability in the spectral response of the land surface. The phenological response by the vegetation of the land surface as measured by a spectral response at the satellite is indicative of both a spatial and temporal response to changes in the seasonal and interannual climate cycles. From this perspective, the spatial boundary between classes of phenological response by vegetation in the southern basin varies greatest to the seasonal climate cycles associated with the onset of the dry season (January or February) than to the seasonal climate cycles of the wet season (June or July). Models that seek to link phenological change of vegetation to biogeochemical responses observed in either the timing or location of organic matter accumulation will need to link spatially explicit phenological behavior with temporally explicit attributes of seasonal climate change.

Acknowledgments. The authors wish to acknowledge the contribution of Dr. Bruce Nelson in the very early stages of reference area selection for this project, Dr. C. J. Tucker for his assistance in the construction of the time series dataset, and the Large-Scale Biosphere-Atmosphere Experiment (LBA) research initiative.

\section{References}

Cressie, N. A., 1991: Statistics for Spatial Data. John Wiley and Sons, 895 pp.

Dirmeyer, P. A., and L. Tan, 2001: A multi-decadal global land-surface data set of state variables and fluxes. COLA Tech. Rep. 102, 43 pp. [Available from the Center for Ocean-LandAtmosphere Studies, 4041 Powder Mill Rd., Suite 302, Calverton, MD 20705.]

Donoho, D. L., 1994: Smooth wavelet decomposition with blocky coefficient kernels. Recent Advances in Wavelet Analysis, L. L. Schumaker and G. Webb, Eds., Academic Press, 1-43.

Dunning, J. B., B. J. Danielson, and H. R. Pulliam, 1992: Processes that effect populations in complex landscapes. Oikos, 65, 169-175.

Goward, S. N., S. Turner, D. G. Dye, and S. Liang, 1994: The University of Maryland improved global vegetation index product. Int. J. Remote Sens., 15, 3365-3395.

Justice, C. O., J. R. G. Townsend, B. N. Holben, and C. J. Tucker, 1985: Analysis of the phenology of global vegetation using meteorological satellite data. Int. J. Remote Sens., 6, 1271-1318.

Laurance, W. F., and G. B. Williamson, 2001: Positive feedbacks among forest fragmentation, drought, and climate change in the Amazon. Conserv. Biol., 15, 1529-1535.

Malingreau, J. P., and C. J. Tucker, 1988: Large scale deforestation in the Southeastern Amazon Basin of Brazil. Ambio, 17, 49-55.

McGarigal, K., and B. J. Marks, 1994: Fragstats: Spatial pattern analysis program for quantifying landscape structure. General Tech. Rep. PNW-GTR-351, Oregon State University, 67 pp.

Ripley, B. D., 1988: Statistical Inference for Spatial Processes. Cambridge University Press, $148 \mathrm{pp}$.

Sellers, P. J., C. J. Tucker, G. J. Collatz, S. O. Los, C. O. Justice, D. A. Dazlich, and D. A. Randall, 1994: A global $1^{\circ}$ by $1^{\circ}$ NDVI data set for climate studies. Part 2: The generation of global fields of terrestrial biophysical parameters from the NDVI. Int. J. Remote Sens., 15, 35193545. 
Setzer, A. W., and M. C. Pereira, 1991: Amazonia biomass burnings in 1987 and an estimate of their tropospheric emissions. Ambio, 20, 19-22.

Tucker, C. J., B. N. Holben, and T. E. Goff, 1984: Intensive forest clearing in Rondonia Brazil as detected by satellite remote sensing. Remote Sens. Environ., 15 (3), 255-262.

Turner, M. G., R. V. O'Neill, R. H. Gardner, and B. T. Milne, 1989: Effects of changing spatial scale on the analysis of landscape pattern. Landscape Ecol., 3 (3-4), 153-162.

Wiens, J. A., 1989: Spatial scaling in ecology. Funct. Ecol., 3, 385-397.

Earth Interactions is published jointly by the American Meteorological Society, the American Geophysical Union, and the Association of American Geographers. Permission to use figures, tables, and brief excerpts from this journal in scientific and educational works is hereby granted provided that the source is acknowledged. Any use of material in this journal that is determined to be "fair use" under Section 107 or that satisfies the conditions specified in Section 108 of the U.S. Copyright Law (17 USC, as revised by P.IL. 94-553) does not require the publishers' permission. For permission for any other form of copying, contact one of the copublishing societies. 
Copyright of Earth Interactions is the property of American Meteorological Society and its content may not be copied or emailed to multiple sites or posted to a listserv without the copyright holder's express written permission. However, users may print, download, or email articles for individual use. 